\title{
Identification of genes associated with castration-resistant prostate cancer by gene expression profile analysis
}

\author{
CHUI GUO HUANG ${ }^{1}$, FENG XI LI ${ }^{2}$, SONG PAN $^{3}$, CHANG BAO XU ${ }^{1}$, JUN QIANG DAI ${ }^{4}$ and XING HUA ZHAO ${ }^{1}$ \\ ${ }^{1}$ Department of Urology, The Second Affiliated Hospital, Zhengzhou University, Zhengzhou, Henan 450014; ${ }^{2}$ Department \\ of Gastrointestinal Glands Surgery, The First Affiliated Hospital, Guangxi Medical University, Nanning, Guangxi 530000; \\ ${ }^{3}$ Department of Urology, The First Affiliated Hospital, Zhengzhou University, Zhengzhou, Henan 450014; ${ }^{4}$ Department \\ of Neurosurgery, The Second Affiliated Hospital, Lanzhou University, Lanzhou, Gansu 730030, P.R. China
}

Received December 16, 2016; Accepted July 17, 2017

DOI: $10.3892 / \mathrm{mmr} .2017 .7488$

\begin{abstract}
Prostate cancer $(\mathrm{CaP})$ is a serious and common genital tumor. Generally, men with metastatic CaP can easily develop castration-resistant prostate cancer (CRPC). However, the pathogenesis and tumorigenic pathways of CRPC remain to be elucidated. The present study performed a comprehensive analysis on the gene expression profile of CRPC in order to determine the pathogenesis and tumorigenic of CRPC. The GSE33316 microarray, which consisted of 5 non-castrated samples and 5 castrated samples, was downloaded from the gene expression omnibus database. Subsequently, 201 upregulated and 161 downregulated differentially expressed genes (DEGs) were identified using the limma package in $\mathrm{R}$ and those genes were classified and annotated by plugin Mcode of Cytoscape. Gene ontology (GO) and Kyoto Encyclopedia of Genes and Genomes (KEGG) pathway enrichment analyses were performed using Database for Annotation, Visualization and Integrated Discovery and KEGG Orthology Based Annotation System 2.0 online tools to investigate the function of different gene modules. The BiNGO tool was used to visualize the level of enriched GO terms. Protein-protein interaction network was constructed using STRING and analyzed with Cytoscape. In conclusion, the present study determined that aldo-keto reductase 3, cyclin B2, regulator of $\mathrm{G}$ protein signaling 2, nuclear factor of activated T-cells and protein kinase $\mathrm{C}$ a may have important roles in the development of CRPC.
\end{abstract}

\section{Introduction}

Prostate cancer (CaP), as the most common genital neoplasm, holds the highest incidence among men in the majority of

Correspondence to: Dr Xing Hua Zhao, Department of Urology, The Second Affiliated Hospital, Zhengzhou University, 2 Jingba Road, Zhengzhou, Henan 450014, P.R. China

E-mail: xhzhao2006@163.com

Key words: castration-resistant prostate cancer, protein-protein interaction network, differentially expressed genes, module analysis western countries (1). CaP growth is driven by androgens; therefore, the conventional treatment option is to lower the levels of male sex hormones (2). Current treatment strategies for $\mathrm{CaP}$ include surgery, external beam radiotherapy, brachytherapy, chemotherapy and androgen-deprivation therapy (ADT) (2,3). ADT often involves surgically removing the testicles or using drugs to block the androgens from affecting the body (4). Unfortunately, ADT has a limited role in preventing majority of patients progressing to castration-resistant prostate cancer (CRPC) and patients with metastatic prostate cancer progress to resistance of ADT $(4,5)$. However, previous studies have been unable to identity a mainstay cancer therapy. Thus, the mortality of patients with CRPC continues to be very high (6).

Previous studies have investigated novel molecular therapeutic targets in CRPC and the influence that tumor metastasis has on the biological processes. Activation of the androgen receptor (AR) may stimulate tumor progression and proliferation in prostate cancer (7), Koivisto et al (8) determined that the activity of cytochrome P450 (CYP) may increase AR gene amplification and continuous production of testosterone; therefore, the serum androgen levels following castration can still contribute to prostate cancer cell growth and resistance. Seruga et al (9) determined that there are three core genes, including human epidermal growth factor receptor 2, transforming growth factor $\beta$ (TGF- $\beta$ ) and the kinase of SRC family that are able to activate AR transduction pathways without androgen to stimulate. Chaux et al (10) suggested that the loss or mutation of tensin homolog have been implicated in unlike properties of aggressive prostate cancer, including increasing the risk of biochemical relapse, reducing the time to metastasis and increasing the death rate in high-risk cohorts of men. However, the molecular mechanisms with oncogenes or tumor suppressors that modulate the levels of critical proteins remain unclear and their relevance in human disease and therapy require further investigation.

In order to provide novel mechanistic insights associated with possible endogenous metastatic pathways in an androgen-deprived environment, the present study used the data from the gene expression profile available provided by Sun et al (11) that used the microarray of human prostate cancer xenograft model-LuCaP35 to analyze the gene 


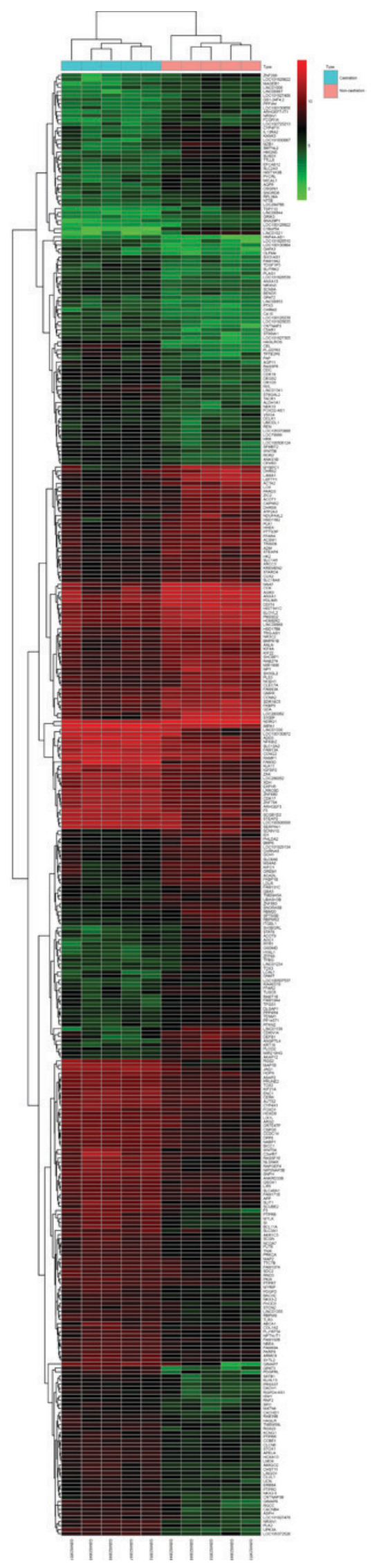

Figure 1. Hierarchical clustering heat maps of the DEGs. The horizontal axis represents sample names, with GSM823845, GSM823846, GSM823847, GSM823851 and GSM823852 being the castration samples, and GSM823844, GSM823848, GSM823849, GSM823850 and GSM823853 the non-castration samples. The vertical axis indicates the clusters of DEGs: Red color stands for an expression level above the mean and green color stands for the expression lower than the mean. DEGs, differentially expressed genes.
Table I. Top 10 upregulated and downregulated genes.

\begin{tabular}{lcc}
\hline A, Upregulated & & \\
\hline Gene symbol & $\operatorname{logFC}$ & Adjusted P-value \\
\hline F3 & 4.863582313 & $2.91 \times 10^{-3}$ \\
HAGLROS & 4.847973534 & $9.32 \times 10^{-6}$ \\
GIMAP7 & 4.839624566 & $4.72 \times 10^{-3}$ \\
CEL & 4.793641494 & $5.79 \times 10^{-4}$ \\
GPAT3 & 4.585119916 & $3.68 \times 10^{-3}$ \\
FAM3D & 4.358475955 & $2.36 \times 10^{-3}$ \\
PDGFRL & 3.929026478 & $1.09 \times 10^{-3}$ \\
RGS2 & 3.899872367 & $3.01 \times 10^{-3}$ \\
UPK3A & 3.868345004 & $5.79 \times 10^{-4}$ \\
SI & 3.843651777 & $7.42 \times 10^{-4}$ \\
\hline
\end{tabular}

B, Downregulated

\begin{tabular}{lcc}
\hline Gene symbol & $\log F C$ & Adjusted P-value \\
\hline MYBPC1 & -4.622857598 & $7.75 \times 10^{-3}$ \\
LAMA1 & -4.215584491 & $1.45 \times 10^{-3}$ \\
S100P & -4.16482084 & $9.77 \times 10^{-3}$ \\
LEFTY1 & -4.151682649 & $8.22 \times 10^{-3}$ \\
DEFB1 & -3.863896718 & $1.59 \times 10^{-3}$ \\
LOX & -3.400271316 & $1.06 \times 10^{-3}$ \\
DHRS2 & -3.381711553 & $2.59 \times 10^{-3}$ \\
CCK & -3.328128227 & $1.53 \times 10^{-3}$ \\
KRT19 & -3.323100902 & $6.53 \times 10^{-3}$ \\
ANGPYL4 & -3.29177361 & $9.74 \times 10^{-3}$
\end{tabular}

FC, fold-change.

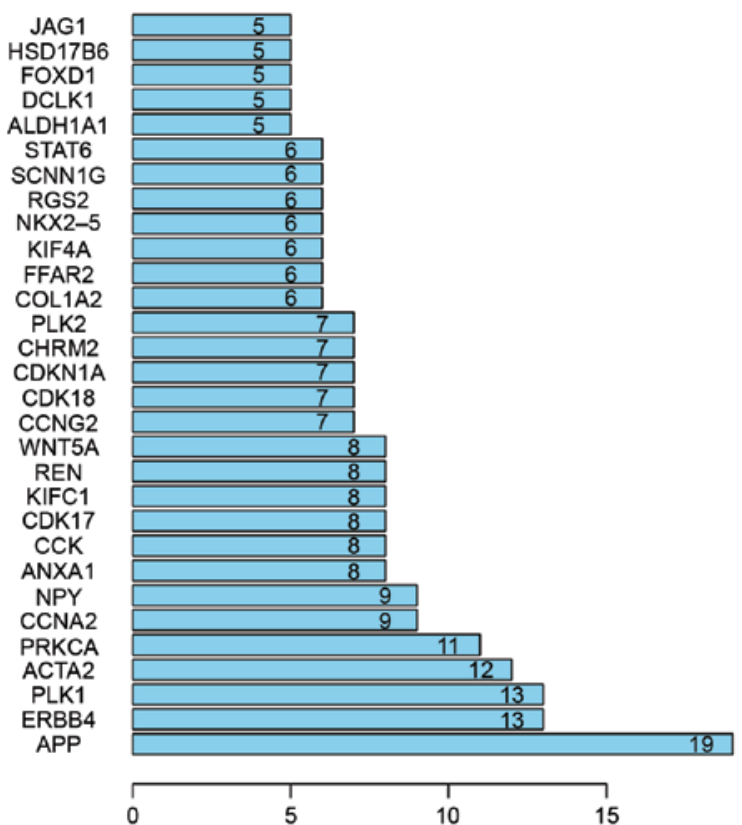

Figure 2. Top 30 hub genes in from the constructed protein-protein interaction network. The horizontal axis represents degree. The vertical axis indicated hub genes. 


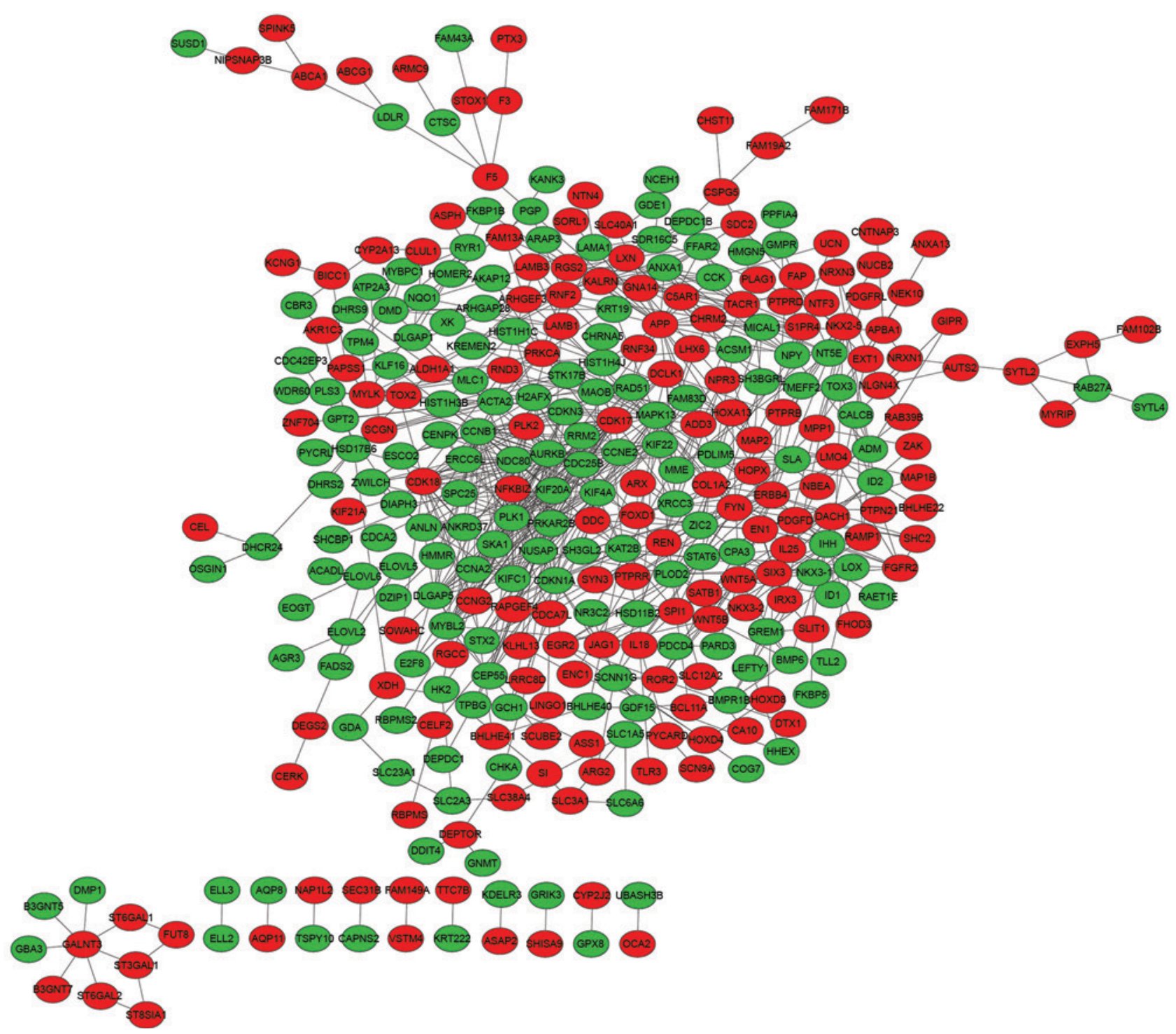

Figure 3. Protein-protein interaction network constructed from the DEGs. Red circles indicate upregulated DEGs and green circles indicate downregulated DEGs. DEGs, differentially expressed genes.

expression changes between 5 non-castrated and 5 castrated men. The current study identified 201 upregulated differentially expressed genes (DEGs) and 161 downregulated DEGs using a stricter threshold of false discovery rate $($ FDR $)<0.05$ and $\mid \log _{2}$ fold-change (FC) $\mid>1.5$. The visual protein-to-protein interaction (PPI) network was constructed using Cytoscape and the modules were identified with the Mcode plugin. The present study used Gene Ontology (GO) and Kyoto Encyclopedia of Genes and Genomes (KEGG) pathway to analyze the potential interactions and functions of DEGs. In conclusion, the present study identified key genes which may have the potential to be biomarkers of tumorigenesis in patients with CRPC.

\section{Materials and methods}

Microarray data. The transcription profile of GSE33316 was downloaded from the National Center for Biotechnology Information Gene Expression Omnibus (GEO) database (www.ncbi.nlm.nih.gov/geo/) (11). The data used a human prostate cancer xenograft model-LuCaP35, included 5 controlled samples from non-castrated men (GSM823844, GSM823848, GSM823849, GSM823850, GSM823853) and 5 samples from castrated men (GSM823845, GSM823846, GSM823847, GSM823851, GSM823852).

Differentially expressed gene analysis. The series matrix file was downloaded and a $\log _{2}$ transformation was performed. All sample data was normalized using the limma package in R software version 3.3.0 (https://www.r-project.org/) (12). The DEGs were obtained with thresholds of $\mid \log \mathrm{FCl}>1.5$ and $\mathrm{P}<0.05$, using linear models and empirical Bayes methods for assessing differential expression in microarray experiments. Finally, a clustering analysis was performed using the DEGs and a heatmap of different groups including castrated and non-castrated was constructed (Fig. 1).

Construction of PPI network and module analysis. In order to predict protein interactions, which include physical and functional associations the present study used the Search Tool 


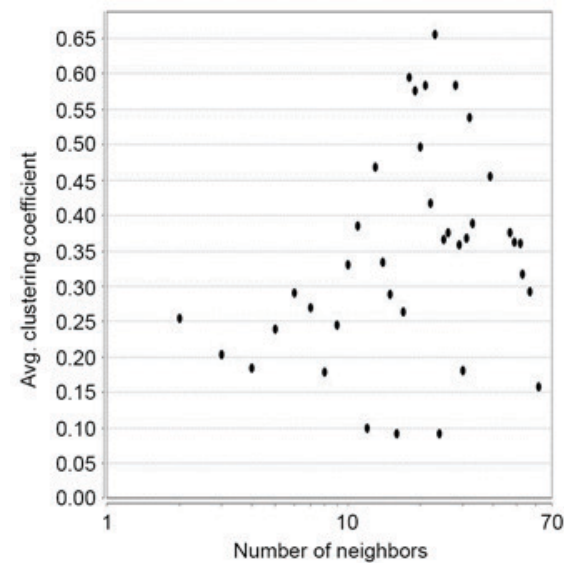

d

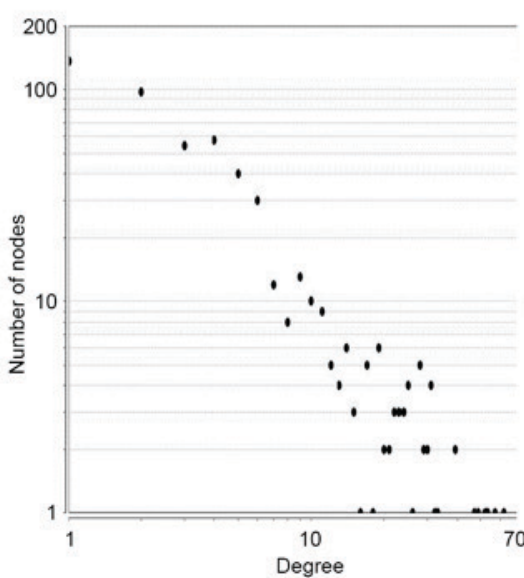

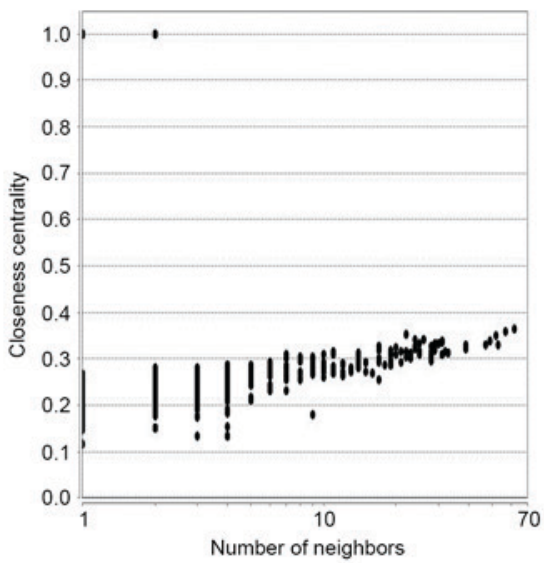

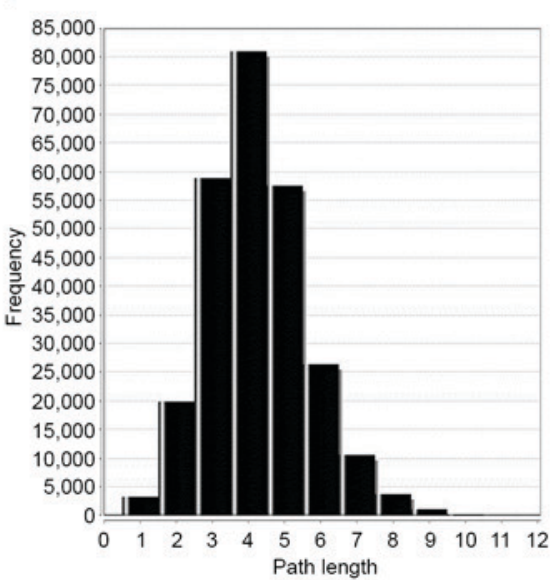

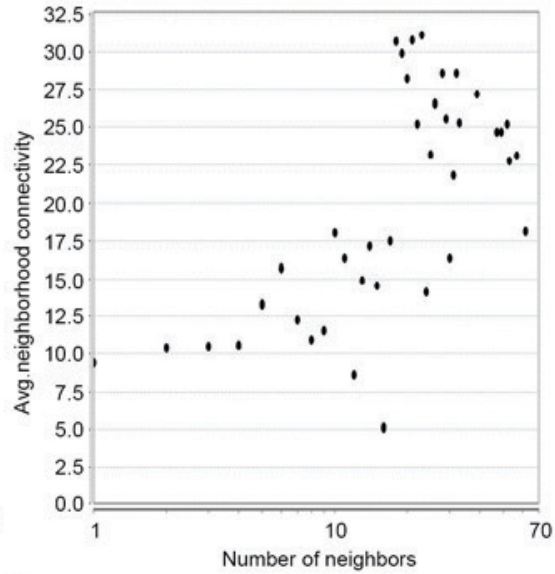

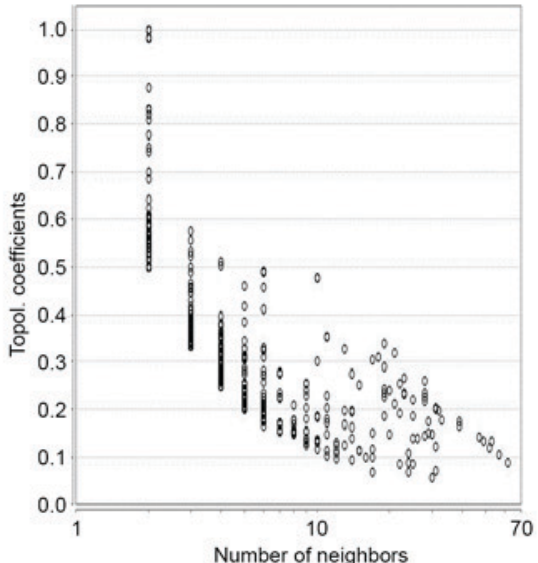

Figure 4. Topology parameters of protein-protein interaction networks. (A) Average clustering coefficient distribution. (B) Closeness centrality. (C) Neighborhood connectivity distribution. (D) Node-degree distribution. (E) Shortest path length distribution. (F) Topological coefficients.

for the Retrieval of Interacting Genes (STRING) to construct the PPI network for DEGs (minimum required interaction score $>0.4$ ) (13). In addition, Cytoscape software version 3.4.0 (http://cytoscape.org/download_old_versions.html) was used for visualization of the PPI networks. Following the construction of the PPI network, a module analysis of the network was performed using the Mcode plugin (degree cut-off $\geq 2$ and the nodes with edges $\geq 2$-core) (14). Additionally, the Network Analyzer was used to compute the basic properties of the PPI network, including average clustering coefficient distribution, closeness centrality, average neighborhood connectivity, node degree distribution, shortest path length distribution, and topological coefficients (15).

GO terms and KEGG pathway analysis. KEGG pathways were investigated by KEGG Orthology Based Annotation System 2.0 online biological tools (16). To analyze the function of DEGs a gene ontology (GO) enrichment analysis was performed using Database for Annotation, Visualization and Integrated Discovery method (17), with a FDR $<0.05$ and count $>2$ as threshold.

BiNGO, is a useful tool to determine which GO categories are statistically overrepresented in a set of genes or a subgraph of a biological network (18), the present study used the visualization of the results of enriched GO terms and the output the predominant functional themes of a given gene set in the GO hierarchy. The findings are presented in a directed acyclic graph.

\section{Results}

Identification of DEGs. Using microarray expression profiling from GEO database, the present study identified the significant DEGs in castrated samples compared with the non-castrated samples. There were 161 downregulated DEGs and 201 upregulated DEGs, respectively accounting for 44.48 and $55.52 \%$ of all DEGs (Fig. 1; Table I). The upregulated genes were more numerous compared with the downregulated genes. A heat map for the expression of the DEGs is presented in Fig. 1. The results of hierarchical clustering analysis indicated that the castrated group and non-castrated group were separated by the clustering the of the DEGs, which indicated that the sectionalization was reasonable and the data may be directly used for further analysis.

Construction of the PPI network using STRING. The STRING database was used to predict the interaction relationship between 362 DEGs (combined score $>0.4$ ). There were 306 nodes and 767 interactions analyzed in the PPI network. The top 30 hub genes are presented in Fig. 2, including amyloid $\beta$ precursor protein (APP), ERBB4, polo like kinase 1 (PLK1), protein kinase $\mathrm{C} \alpha$ (PRKCA), actin, alpha 2, smooth muscle, aorta and cyclin A2. The visualization of the PPI network used Cytoscape and is shown in Fig. 3. 

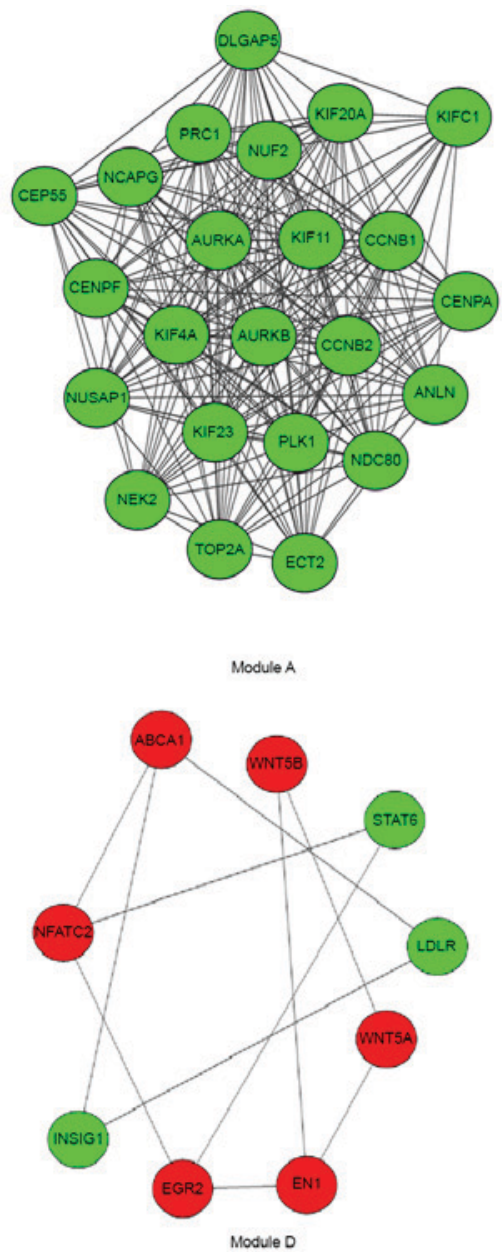
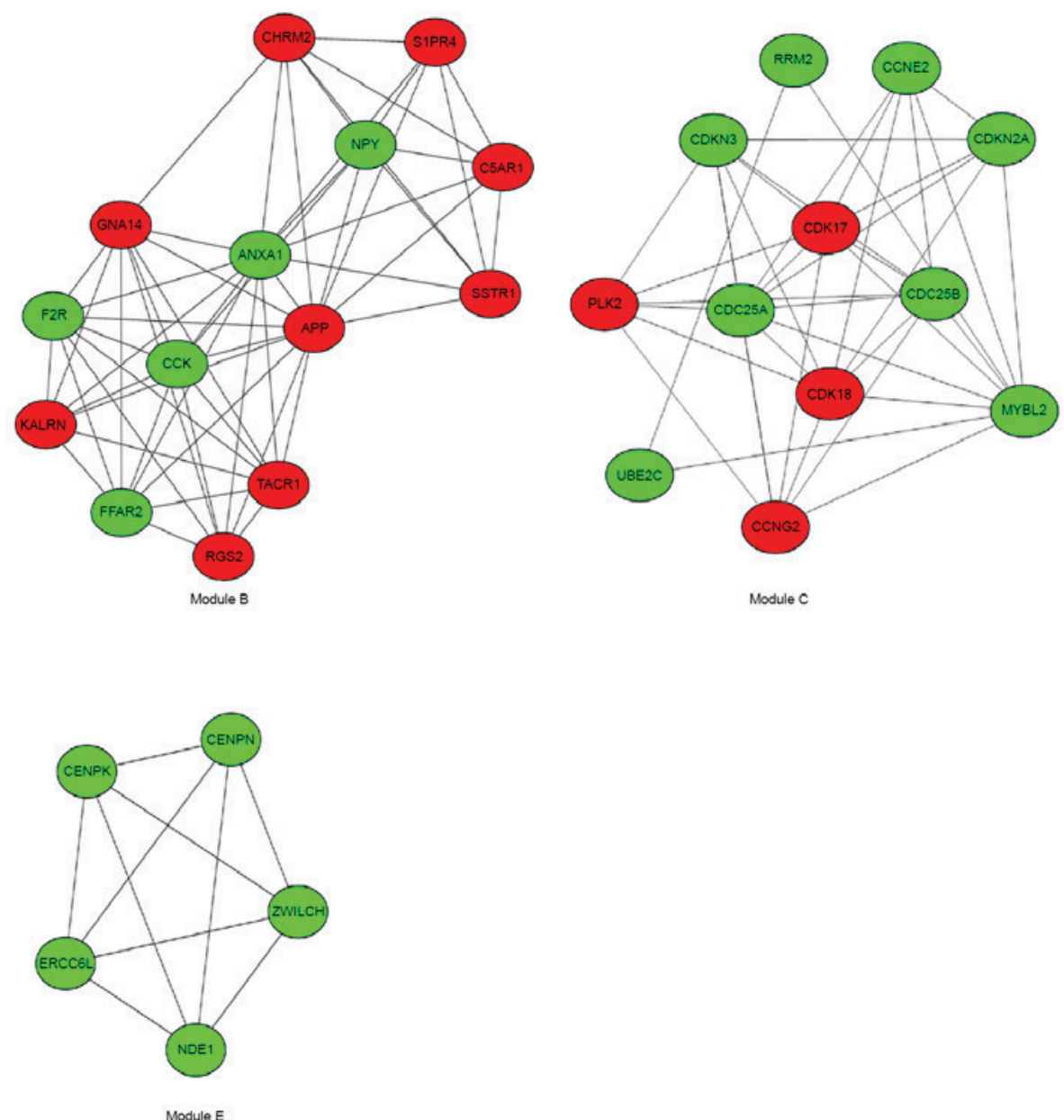

Figure 5. Top 5 modules obtained from the protein-protein interaction network of the differentially expressed genes. The circular and triangular nodes represent proteins (triangular nodes indicate hub proteins) and the grey lines represent interactions. Red circles indicate upregulated genes and green circles indicate downregulated genes.

Network module analysis. The topology parameter of the PPI network was based on the following six properties: i) Average clustering coefficient distribution; ii) closeness centrality; iii) neighborhood connectivity distribution; iv) node degree distribution; v) shortest path length distribution; and vi) topological coefficients (Fig. 4). This analysis revealed that the constructed network is stable and reliable. A total of 10 modules were identified in the PPI network and the top 5 modules were investigated further (Fig. 5), which included 23, 14, 12, 9 and 5 genes (Table II). Additionally, the DEGs in the top 5 modules were enriched in the significant pathways presented in Table III. Module A had 23 nodes and 222 interactions. All the DEGs were downregulated in this module. There were 5 enriched KEGG pathways in this module, which involved cyclin B1 (CCNB1) and CCNB2, PLK1 and aurora kinase A (AURKA).

Module B had 14 nodes and 57 interactions. There were 9 upregulated DEGs, including cholinergic receptor muscarinic 2 (CHRM2), sphingosine-1-phosphate receptor 4 (S1PR4), complement component 5a receptor 1 (C5AR1), somatostatin receptor 1 (SSTR1), APP, tachykinin receptor 1 (TACR1), regulator of G protein signaling 2 (RGS2), G protein subunit $\alpha 14$ and kalirin RhoGEF kinase, and 5 downregulated DEGs, including coagulation factor II thrombin receptor
(F2R), free fatty acid receptor 2, cholecystokinin, annexin A1 and neuropeptide Y. For the DEGs in this module, 6 enriched KEGG pathways were identified. The DEGs involved in these pathways were CHRM2, SSTR1, F2R and TACR1.

Module $\mathrm{C}$ had 12 nodes and 39 interactions. In this module, the number of upregulated genes was reduced compared with the downregulated genes, 4 and 8, respectively. A total of 6 enriched KEGG pathways were identified, which involved cell division cycle 25 , cyclin dependent kinase inhibitor $2 \mathrm{~A}$, cyclin E2 and ribonucleotide reductase regulatory subunit M2.

Module D had 9 nodes and 11 interactions. The number of upregulated genes was higher compared with the downregulated genes, 8 and 4,respectively. There were 10 enriched KEGG pathways identified in this module. The DEGs, including Wnt family member 5A (WNT5A) and WNT5B, signal transducer and activator of transcription 6 (STAT6), nuclear factor of activated T-cells 2 and early growth response 2 (EGR2) were screened in these pathways.

Module E had 5 nodes and 10 interactions. It consists of downregulated genes, nudE neurodevelopment protein 1, centromere protein $\mathrm{K}$, zwilch kinetochore protein, centromere protein $\mathrm{N}$ and ERCC excision repair 6 like, spindle assembly checkpoint helicase; however, no significantly enriched KEGG pathways were identified in this module. 
Table II. The modules of networks.

\begin{tabular}{lccc}
\hline Module name & Nodes & Edges & Cluster scores \\
\hline A & 23 & 222 & 20.182 \\
B & 14 & 57 & 8.769 \\
C & 12 & 39 & 7.091 \\
D & 9 & 11 & 5.75 \\
E & 5 & 10 & 5.00 \\
\hline
\end{tabular}

Functional analysis. Three types of functions including the biological processes (BP), cellular components (CC) and molecular functions (MF) of DEGs were annotated and classified by a comprehensive GO analysis. The present study identified a total of $26 \mathrm{GO}$ terms based on the DEGs of modules with a FDR $<0.05$ and count $>2$ as threshold and then these terms were sorted by P-value. From the GO terms, 16 enriched GO terms in BP, 7 enriched GO terms in $\mathrm{CC}$ and 3 enriched GO terms enriched in MF were identified (Fig. 6; Table IV). An acyclic graph was constructed and directed using the BiNGO tool to depict the visual interactions of functions based on the enrichment levels of the GO terms (Fig. 7).

\section{Discussion}

$\mathrm{CaP}$ is the most common non-cutaneous carcinoma among elderly men, and $\sim 180,890$ men have been estimated to have been diagnosed in the USA by 2016 (1). ADT as the primary therapeutic strategy for advanced stage of prostate carcinoma has been the conventional treatment since 1942; however, the disease frequently relapses and progresses into CRPC with adaptive responses of androgen receptors $(4,7,19)$. Therefore, the present study aimed to outline potential prognostic biomarkers or therapeutic targets for CRPC.

In the present study, 362 DEGs were identified from the gene expression profile analysis. Due to gene-gene interactions it is more accurate to predict the associations of protein functions compared with a single gene and the present study identified 306 interacting genes and constructed a PPI network using the STRING online tool. Accordingly, to show all DEGs, a network of PPI interactions was generated. The DEGs in the PPI network exhibited higher enrichment, indicating a higher degree of modularization; therefore, the present study divided the DEGs into 10 modules for investigating the interactions using Mcode and the top 5 modules were selected for further investigation. Additionally, the GO analysis demonstrated that the function of DEGs in different cluster modules were associated with androgen metabolism and cell cycle. The KEGG pathway analysis was performed to identify the altered pathways in the functional modules. Finally, aldo-keto reductase 3 (AKR1C3), CCNB2, RGS2, nuclear factor of activated T-cells (NFATc2) and PRKCA were significantly enriched in CRPC development.

AKR1C3 is a member of aldo-keto reductase superfamily, which includes $\sim 40$ types of proteins and enzymes. These enzymes use NADH or NADPH as cofactors to convert aldehydes and ketones into the relevant alcohols with catalyzed reaction (20). Byrns et al (21) showed that AKR1C3 overexpression was associated with the high risk of breast and ovarian cancer. Azzarello et al (22) determined that the high expression of AKR1C3 may be a predictor for poor prognosis in patients with renal cell carcinoma, papillary urothelial carcinoma, and Wilms' tumor. The GO enrichment performed by the present study demonstrated that AKR1C3 was associated with biological processes and molecular functions, such as cellular process, oxidation reduction, steroid dehydrogenase activity and testosterone $17-\beta$-dehydrogenase activity. Therefore, it possible that AKR1C3 is an important enzyme mediated in the progression of $\mathrm{CaP}$ into the development of CRPC. Additionally, AKR1C3 is the upregulated DEG in this gene expression profile; therefore, knocking-down the expression of AKR1C3 may lower the androgen sensitivity of CRPC cells.

CCNB2 was identified as the core gene of module A. CCNB2 is a B-type cyclin of the cyclin family. CCNB2 and $\mathrm{B} 1$, associated with $\mathrm{p} 34 \mathrm{cdc} 2 /$ cyclin B complex, are essential components of the cell cycle regulatory machinery (23). In the module A, enriched KEGG pathways revealed that CCNB2 was associated with pathways, including cell cycle, FoxO and p53 signaling pathways. In addition, through comparison of functions between GO enrichments and the DEGs, the present study identified that CCNB2, a key enzyme mediating in the progress of oxidation reduction, was associated with the cell cycle. The epithelial-mesenchymal transition (EMT) has a crucial role in the developmental process during which prostate cancer cells acquire migrating and invasive phenotype $(11,24)$. Additionally, EMT may promote cancer metastasis and mediate the resistance to ADT in prostate cancer (25). Shiota et al (26) identified TGF- $\beta$ as an EMT inducer that leads AR to acquire castration resistance. A previous study revealed that in the FoxO signaling pathway, CCNB2 may bind TGF- $\beta$-RII and may be involved in transforming growth factor $b$-mediated cell cycle control (27). Therefore, the present study suggested that CCNB2 may be a co-regulator of TGF- $\beta$, mediating the development of tumor metastasis and therapeutic resistance; therefore, the effective treatment strategy for CRPC may involve altering the expression of CCNB2. CCNB2 has interactive associations with PLK1 and AURKA in module A, revealing that CCNB2 may also be involved in CRPC by mediating PLK1 and AURKA.

$\mathrm{G}$-protein coupled receptors are important in the physiology of the prostate; therefore, they may be a potential alternative or combinatorial therapeutic targets in the growth processes of tumor cells and AR-mediated signaling pathways (28). The present study identified RGS2 as a differentially expressed gene enriched in the cAMP signaling pathway of module B. In addition, RGS2 has a crucial role in cell cycle according to the GO enrichment analysis. The cell cycle is a succession of events which take place at the cellular level resulting in division and duplication (29) and thus it indicated that RGS2 may associate with the division and metabolism in tumor cells. The expression of RGS2 is upregulated in the castrated samples of the present study, its overexpression may induce a change for the sensitivity of AR. Therefore, a knockdown of the expression of RGS2 may suppress the growth of tumor cells and block the progression from androgen dependence to castration resistance. In 
Table III. KEGG pathways of the modules in the present study.

A, Module A

\begin{tabular}{llr}
\hline KEGG pathways & \multicolumn{1}{c}{ Genes } & P-value \\
\hline $\begin{array}{l}\text { hsa04114: Oocyte meiosis } \\
\text { hsa04914: Progesterone-mediated }\end{array}$ & CCNB2, CCNB1, PLK1, AURKA & $1.59 \times 10^{-6}$ \\
$\begin{array}{l}\text { oocyte maturation } \\
\text { hsa04110: Cell cycle }\end{array}$ & CCNB2, CCNB1, PLK1 & $4.49 \times 10^{-5}$ \\
hsa04068: FoxO signaling pathway & CCNB2, CCNB1, PLK1 \\
hsa04115: p53 signaling pathway & CCNB2, CCNB1, PLK1 & $8.87 \times 10^{-5}$ \\
& CCNB2, CCNB1 & $1.11 \times 10^{-4}$ \\
& & $1.10 \times 10^{-3}$ \\
\hline
\end{tabular}

B, Module B

\begin{tabular}{llr}
\hline KEGG pathways & \multicolumn{1}{c}{ Genes } & P-value \\
\hline $\begin{array}{l}\text { hsa04080: Neuroactive ligand- } \\
\text { receptor interaction }\end{array}$ & CHRM2, SSTR1, F2R, TACR1, C5AR1, S1PR4 & $5.31 \times 10^{-6}$ \\
hsa04024: cAMP signaling pathway & CHRM2, SSTR1, NPY, RGS2, FFAR2 & $1.70 \times 10^{-5}$ \\
hsa04020: Calcium signaling pathway & GNA14, CHRM2, TACR1, F2R & $2.02 \times 10^{-4}$ \\
hsa04610: Complement and coagulation & C5AR1, F2R & $7.29 \times 10^{-3}$ \\
cascades & & $4.61 \times 10^{-3}$ \\
hsa04810: Regulation of actin cytoskeleton & CHRM2, F2R & $1.03 \times 10^{-2}$ \\
hsa04151: PI3K-Akt signaling pathway & CHRM2, F2R & \\
\hline
\end{tabular}

C, Module C

KEGG pathways

Genes

P-value

hsa04115: p53 signaling pathway

RRM2, CCNG2, CDKN2A, CCNE2

$5.94 \times 10^{-6}$

hsa04110: Cell cycle

CDC25B, CDKN2A, CDC25A, CCNE2

$5.46 \times 10^{-5}$

hsa05206: MicroRNAs in cancer

CDC25B, CDKN2A, CDC25A, CCNE2

$1.44 \times 10^{-3}$

hsa04914: Progesterone-mediated

CDC25A, CDC25B

$1.14 \times 10^{-2}$

oocyte maturation

hsa04068: FoxO signaling pathway

PLK2, CCNG2

$2.05 \times 10^{-2}$

hsa05203: Viral carcinogenesis

CDKN2A, CCNE2

$4.42 \times 10^{-2}$

D, Module D

KEGG pathways

hsa05166: HTLV-I infection

hsa04310: Wnt signaling pathway

hsa05161: Hepatitis B

hsa04360: Axon guidance

hsa05217: Basal cell carcinoma

hsa04916: Melanogenesis

hsa04550: Signaling pathways regulating

pluripotency of stem cells

hsa04390: Hippo signaling pathway

hsa04150: mTOR signaling pathway

hsa05205: Proteoglycans in cancer
Genes

P-value

EGR2, WNT5A, WNT5B, NFATC2

$1.11 \times 10^{-3}$

WNT5A, WNT5B, NFATC2

$2.08 \times 10^{-3}$

EGR2, STAT6, NFATC2

$2.20 \times 10^{-3}$

WNT5A, WNT5B, NFATC2

$3.70 \times 10^{-3}$

WNT5A, WNT5B

$4.41 \times 10^{-3}$

WNT5A, WNT5B

$1.36 \times 10^{-2}$

WNT5A, WNT5B

$2.59 \times 10^{-2}$

WNT5A, WNT5B

$3.00 \times 10^{-2}$

WNT5A, WNT5B

$3.01 \times 10^{-2}$

WNT5A, WNT5B 


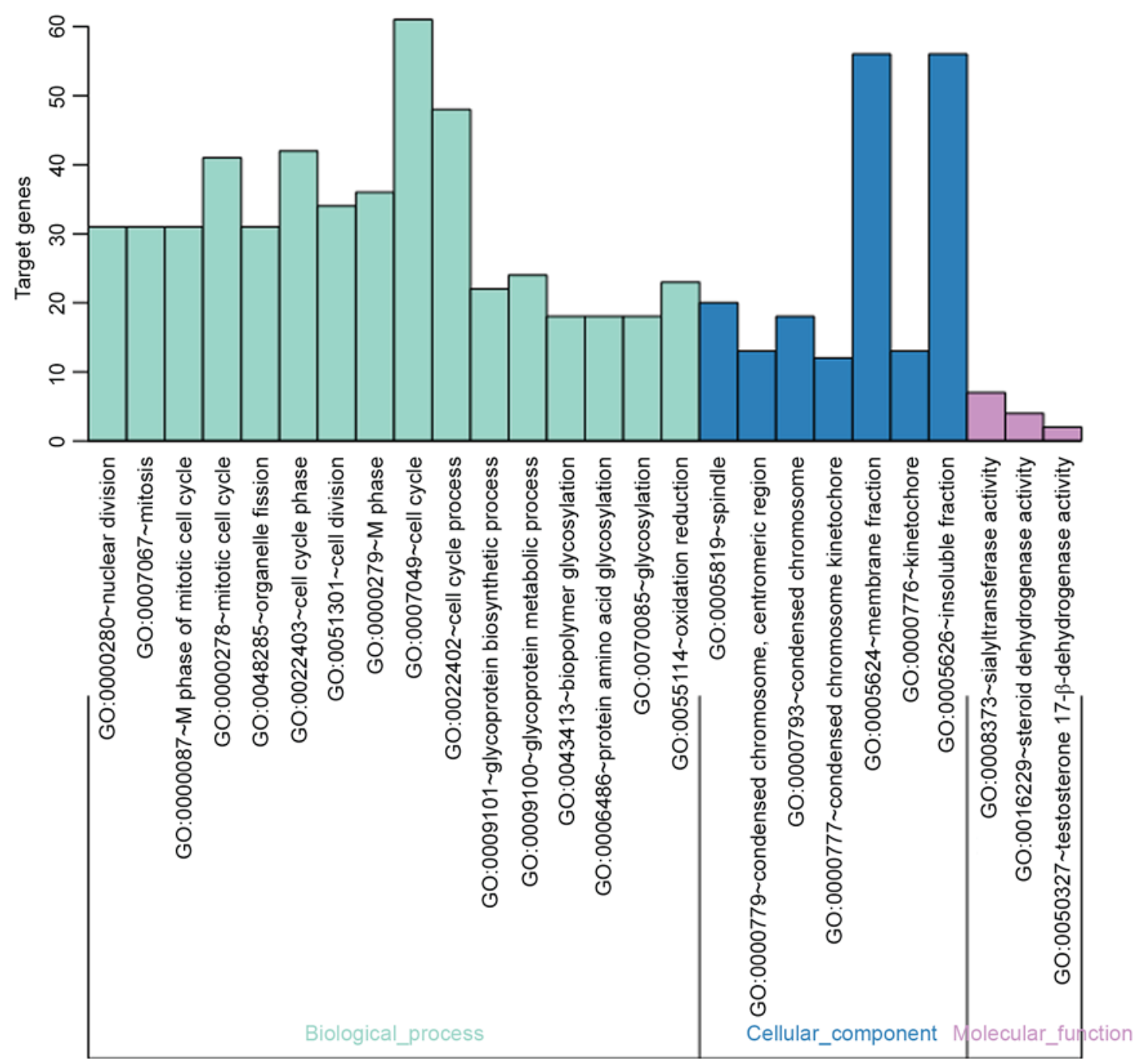

Figure 6. Biological processes, cellular components and molecular functions of differentially expressed genes were annotated and classified using GO analysis. The horizontal axis indicates the names of GO terms. GO, gene ontology.

module B, RGS2 has interactive associations with CHRM2, SSTR1, F2R, TACR1, C5AR1 and S1PR4, indicating that RGS2 may also be involved in CRPC by mediating these genes.

NFATc2, a member of NFAT family, was identified as a central regulator in activation of gene transcription during immune response (30). However, the present study determined that NFATc2 was a core gene enriched in the Wnt signaling pathway of module D for prostate cancer. NFATc2 may activate in response to the Wnt signaling pathway stimulation and activate $\beta$-catenin expression (31). Additionally, $\beta$-catenin is a cofactor of AR that may amplify AR signaling to regulate androgen synthesis (32). Therefore, it was hypothesized that NFATc2 may be a key regulator correlated with CRPC. In addition, NFATc2 has interactive associations with EGR2, STAT6, Wnt5A and Wnt5B in module D, revealing that NFATc2 may also be involved in CRPC by mediating these genes.

PRKCA is a member of protein kinase C (PKC) family that may be activated by calcium and the second messenger diacylglycerol (33). Using the PPI network constructed by the present study PRKCA was identified as a hub gene with 11 interactions. Tirado et al (34) determined that
PRKCA may modulate caveolin-1 to promote resistance to chemotherapy-induced apoptosis in Ewing's sarcoma. Lønne et al (35) showed that PKCa expression may be a marker for breast cancer aggressiveness. In addition, Lichner et al (36) demonstrated that PRKCA may reduce cell proliferation and migration of prostate cancer. However, to the best of our knowledge, there is no experimental study reporting PRKCA to be associated with CRPC. Nonetheless, the PPI network constructed in the present study has indicated that PRKCA may have interactive associations with CRPC, these findings require conformation by future studies.

In summary, based on the gene expression profile analysis in GEO database, the present study identified the DEGs between non-castrated and castrated samples. There were 362 DEGs identified by comprehensive bioinformatics analysis, including AKR1C3, CCNB2, RGS2, NFATc2 and PRKCA, which may have a fundamental role in the development of CRPC and predicted to be involved in the cell cycle pathway. Those findings may be potential biomarkers for the exploration of the biological mechanisms of CRPC and may be used as potential targets for therapeutic intervention or diagnosis of CRPC. 
Table IV. Enriched GO functions for the DEGs.

\begin{tabular}{|c|c|c|c|c|}
\hline GO Term & Function & Count & FDR & Examples of DEGs \\
\hline GO:0000280 & Nuclear division & 31 & $3.83 \times 10^{-6}$ & $\begin{array}{l}\text { CCNB1, CCNB2, PLK1, } \\
\text { AURKB, CCNG2 }\end{array}$ \\
\hline GO:0007067 & Mitosis & 31 & $3.97 \times 10^{-6}$ & $\begin{array}{l}\text { KIF } 23, \text { CCNA2, CCNB2, } \\
\text { PLK1, CDCA2 }\end{array}$ \\
\hline GO:0000087 & $\begin{array}{l}\text { M phase of mitotic } \\
\text { cell cycle }\end{array}$ & 31 & $5.90 \times 10^{-6}$ & $\begin{array}{l}\text { KIF23, KIFC1, KIF22, } \\
\text { NEK2, HAUS1 }\end{array}$ \\
\hline GO:0000278 & Mitotic cell cycle & 41 & $8.90 \times 10^{-6}$ & $\begin{array}{l}\text { CDKN1A, CCNB2, PLK2, } \\
\text { PLK1, RNF2 }\end{array}$ \\
\hline GO:0048285 & Organelle fission & 31 & $9.98 \times 10^{-6}$ & $\begin{array}{l}\text { NDC } 80, \text { UBE2C, CDC25A, } \\
\text { CCNB1, CCNB2 }\end{array}$ \\
\hline GO:0022403 & Cell cycle phase & 42 & $7.08 \times 10^{-5}$ & $\begin{array}{l}\text { BCAT1, KIF23, KIF22, } \\
\text { KIFC1, PRC1 }\end{array}$ \\
\hline GO:0051301 & Cell division & 34 & $9.05 \times 10^{-5}$ & $\begin{array}{l}\text { CCNE2, SPC25, NDE1, } \\
\text { CDKN2A, NCAPG }\end{array}$ \\
\hline GO:0000279 & M phase & 36 & $1.25 \times 10^{-4}$ & $\begin{array}{l}\text { CCNA2, ERCC6L, KIF11, } \\
\text { DLGAP5, NUF2 }\end{array}$ \\
\hline GO:0007049 & Cell cycle & 61 & $4.66 \times 10^{-4}$ & $\begin{array}{l}\text { CCNB2, PLK2, RGS2, } \\
\text { MAPK13, PLK1 }\end{array}$ \\
\hline GO:0022402 & Cell cycle process & 48 & $1.36 \times 10^{-3}$ & $\begin{array}{l}\text { BCAT1, KIF23, KIF22, } \\
\text { KIFC1, PRC1 }\end{array}$ \\
\hline GO:0009101 & $\begin{array}{l}\text { Glycoprotein } \\
\text { biosynthetic process }\end{array}$ & 22 & $1.56 \times 10^{-3}$ & $\begin{array}{l}\text { GALNT3, ST6GAL1, } \\
\text { ST6GAL2, LDLR, FUT8 }\end{array}$ \\
\hline GO:0009100 & $\begin{array}{l}\text { Glycoprotein } \\
\text { metabolic process }\end{array}$ & 24 & $7.23 \times 10^{-3}$ & $\begin{array}{l}\text { GALNT3, ST6GAL1, } \\
\text { ST8SIA1, CHST3, ABCG1 }\end{array}$ \\
\hline GO:0043413 & $\begin{array}{l}\text { Biopolymer } \\
\text { glycosylation }\end{array}$ & 18 & $1.72 \times 10^{-2}$ & $\begin{array}{l}\text { GALNT3, ST6GAL1, } \\
\text { ABCG1, MPDU1, B3GNT3 }\end{array}$ \\
\hline GO:0006486 & $\begin{array}{l}\text { Protein amino acid } \\
\text { glycosylation }\end{array}$ & 18 & $1.73 \times 10^{-2}$ & $\begin{array}{l}\text { COG7, ST3GAL4, B3GNT7, } \\
\text { ST3GAL6, MPDU1 }\end{array}$ \\
\hline GO:0070085 & Glycosylation & 18 & $1.73 \times 10^{-2}$ & $\begin{array}{l}\text { GALNT3, ST6GAL1, } \\
\text { ST6GAL2, ALG1, ST3GAL1 }\end{array}$ \\
\hline GO:0055114 & Oxidation reduction & 23 & $3.15 \times 10^{-2}$ & $\begin{array}{l}\text { DHRS9, ACADL, GMPR, } \\
\text { ALDH1A1, AKR1C3 }\end{array}$ \\
\hline
\end{tabular}

B, Cellular components

\begin{tabular}{|c|c|c|c|c|}
\hline Term & Function & Count & FDR & Examples of DEGs \\
\hline GO:0005819 & Spindle & 20 & $5.53 \times 10^{-3}$ & $\begin{array}{l}\text { KIF23, NDE1, APP, } \\
\text { PLK1, SKA1 }\end{array}$ \\
\hline GO:0000779 & $\begin{array}{l}\text { Condensed chromosome, } \\
\text { centromeric region }\end{array}$ & 13 & $1.02 \times 10^{-2}$ & $\begin{array}{l}\text { CENPN, NUF2, } \\
\text { CENPF, NDC } 80, \text { AURKB }\end{array}$ \\
\hline GO:0000793 & Condensed chromosome & 18 & $1.36 \times 10^{-2}$ & $\begin{array}{l}\text { CENPN, NEK2, SKA1, } \\
\text { CENPF, CHEK1, }\end{array}$ \\
\hline GO:0000777 & $\begin{array}{l}\text { Condensed chromosome } \\
\text { kinetochore }\end{array}$ & 12 & $1.61 \times 10^{-2}$ & $\begin{array}{l}\text { SPC25, CENPN, NDE1, } \\
\text { CENPA, NUF2, }\end{array}$ \\
\hline GO:0005624 & Membrane fraction & 56 & $4.37 \times 10^{-2}$ & $\begin{array}{l}\text { NPR3, RGS16, CCNB2, } \\
\text { APP, CHRM2 }\end{array}$ \\
\hline GO:0000776 & Kinetochore & 13 & $4.48 \times 10^{-2}$ & $\begin{array}{l}\text { KIF22, CENPN, NUF2, } \\
\text { CENPF, CENPK }\end{array}$ \\
\hline GO:0005626 & Insoluble fraction & 56 & $4.12 \times 10^{-2}$ & $\begin{array}{l}\text { CYP2J2, CADM1, CCNB2, } \\
\text { NPR3, RGS16 }\end{array}$ \\
\hline
\end{tabular}


Table IV. Continued.

C, Molecular functions

\begin{tabular}{llcll}
\hline Term & \multicolumn{1}{c}{ Function } & Count & FDR & Examples of DEGs \\
\hline GO:0008373 & Sialyltransferase activity & 7 & $4.14 \times 10^{-2}$ & $\begin{array}{l}\text { ST6GAL1, ST6GAL2, } \\
\text { ST3GAL4, ST3GAL6 } \\
\text { GO:0016229 }\end{array}$ \\
$\begin{array}{llll}\text { Steroid dehydrogenase } \\
\text { activity } \\
\text { Testosterone 17-beta- } \\
\text { dehydrogenase activity }\end{array}$ & 4 & $1.89 \times 10^{-2}$ & $\begin{array}{l}\text { AKR } 3 \text {, HSD11B2, } \\
\text { DHRS9, HSD17B6 } \\
\text { AKR1C3, HSD17B6 }\end{array}$ \\
\end{tabular}

GO, gene ontology; FDR, false discovery rate; DEGs, differentially expressed genes.

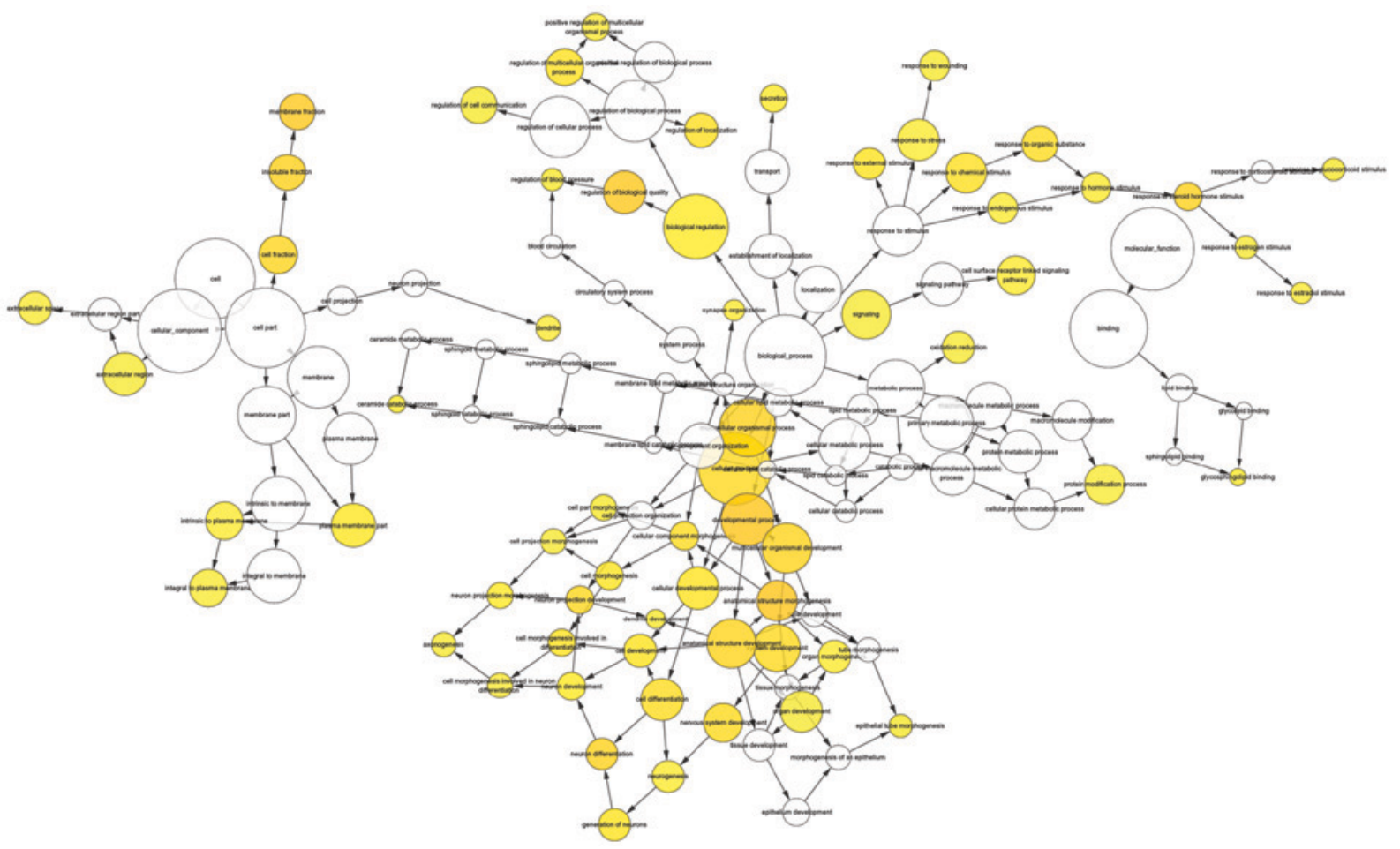

Figure 7. Directed acyclic graph based on the enrichment degree of GO terms. Color depth represents the degree of GO terms enrichment. GO, gene ontology.

However, the primary limitation of the present study is that as the DEGs remain to be verified by experiments; therefore, further analyses are required to determine the mechanisms in process of malignant progression in $\mathrm{CaP}$. Future studies will aim to use polymerase chain reaction or western blotting to verify expression levels of the key genes in samples between non-castrated and castrated men.

\section{References}

1. Siegel RL, Miller KD and Jemal A: Cancer statistics, 2016. CA Cancer J Clin 66: 7-30, 2016.

2. Guinney J, Wang T, Laajala TD, Winner KK, Bare JC, Neto EC, Khan SA, Peddinti G, Airola A, Pahikkala T, et al: Prediction of overall survival for patients with metastatic castration-resistant prostate cancer: Development of a prognostic model through a crowdsourced challenge with open clinical trial data. Lancet Oncol 18: 132-142, 2017.
3. Schmid S, Omlin A, Blum D, Strasser F, Gillessen S and Rothermundt C: Assessment of anticancer-treatment outcome in patients with metastatic castration-resistant prostate cancer-going beyond PSA and imaging, a systematic literature review. Ann Oncol 26: 2221-2247, 2015.

4. Chandrasekar T, Yang JC, Gao AC and Evans CP: Mechanisms of resistance in castration-resistant prostate cancer (CRPC). Transl Androl Urol 4: 365-380, 2015.

5. Damber JE and Aus G: Prostate cancer. Lancet 371: 1710-1721, 2008.

6. Ferlay J, Steliarova-Foucher E, Lortet-Tieulent J, Rosso S, Coebergh JW, Comber H, Forman D and Bray F: Cancer incidence and mortality patterns in Europe: Estimates for 40 countries in 2012. Eur J Cancer 49: 1374-1403, 2013.

7. McCrea E, Sissung TM, Price DK, Chau CH and Figg WD: Androgen receptor variation affects prostate cancer progression and drug resistance. Pharmacol Res 114: 152-162, 2016.

8. Koivisto P, Kononen J, Palmberg C, Tammela T, Hyytinen E, Isola J, Trapman J, Cleutjens K, Noordzij A, Visakorpi T and Kallioniemi OP: Androgen receptor gene amplification: A possible molecular mechanism for androgen deprivation therapy failure in prostate cancer. Cancer Res 57: 314-319, 1997. 
9. Seruga B, Ocana A and Tannock IF: Drug resistance in metastatic castration-resistant prostate cancer. Nat Rev Clin Oncol 8: 12-23, 2011.

10. Chaux A, Peskoe SB, Gonzalez-Roibon N, Schultz L, Albadine R, Hicks J, De Marzo AM, Platz EA and Netto GJ: Loss of PTEN expression is associated with increased risk of recurrence after prostatectomy for clinically localized prostate cancer. Mod Pathol 25: 1543-1549, 2012

11. Sun Y, Wang BE, Leong KG, Yue P, Li L, Jhunjhunwala S, Chen D, Seo K, Modrusan Z, Gao WQ, et al: Androgen deprivation causes epithelial-mesenchymal transition in the prostate: Implications for androgen-deprivation therapy. Cancer Res 72 : 527-536, 2012.

12. Gentleman R, Carey V, Huber W, Irizarry R and Dudoit S, (eds): Bioinformatics and computational biology solutions using $R$ and Bioconductor. Vol. 746718470. Springer, New York, NY, 2005.

13. Franceschini A, Szklarczyk D, Frankild S, Kuhn M, Simonovic M, Roth A, Lin J, Minguez P, Bork P, von Mering C and Jensen LJ: STRING v9.1: Protein-protein interaction networks, with increased coverage and integration. Nucleic Acids Res 41 (Database issue): D808-D815, 2013.

14. Saito R, Smoot ME, Ono K, Ruscheinski J, Wang PL, Lotia S, Pico AR, Bader GD and Ideker T: A travel guide to Cytoscape plugins. Nat Methods 9: 1069-1076, 2012.

15. Assenov Y, Ramírez F, Schelhorn SE, Lengauer $T$ and Albrecht M: Computing topological parameters of biological networks. Bioinformatics 24: 282-284, 2008.

16. Xie C, Mao X, Huang J, Ding Y, Wu J, Dong S, Kong L, Gao G, Li CY and Wei L: KOBAS 2.0: A web server for annotation and identification of enriched pathways and diseases. Nucleic Acids Res 39 (Web Server issue): W316-W322, 2011.

17. Huang da W, Sherman BT and Lempicki RA: Systematic and integrative analysis of large gene lists using DAVID bioinformatics resources. Nat Protoc 4: 44-57, 2009

18. Maere S, Heymans K and Kuiper M: BiNGO: A cytoscape plugin to assess overrepresentation of gene ontology categories in biological networks. Bioinformatics 21: 3448-3449, 2005.

19. Merseburger AS, Alcaraz A and von Klot CA: Androgen deprivation therapy as backbone therapy in the management of prostate cancer. Onco Targets Ther 9: 7263-7274, 2016.

20. Penning TM, Burczynski ME, Jez JM, Hung CF, Lin HK, Ma H, Moore M, Palackal N and Ratnam K: Human 3alpha-hydroxysteroid dehydrogenase isoforms (AKR1C1-AKR1C4) of the aldo-keto reductase superfamily: Functional plasticity and tissue distribution reveals roles in the inactivation and formation of male and female sex hormones. Biochem J 351: 67-77, 2000.

21. Byrns MC, Duan L, Lee SH, Blair IA and Penning TM: Aldo-keto reductase $1 \mathrm{C} 3$ expression in MCF-7 cells reveals roles in steroid hormone and prostaglandin metabolism that may explain its over-expression in breast cancer. J Steroid Biochem Mol Biol 118: 177-187, 2010.
22. Azzarello JT, Lin HK, Gherezghiher A, Zakharov V, Yu Z, Kropp BP, Culkin DJ, Penning TM and Fung KM: Expression of AKR1C3 in renal cell carcinoma, papillary urothelial carcinoma, and Wilms' tumor. Int J Clin Exp Pathol 3: 147-155, 2009.

23. Bellanger S, de Gramont A and Sobczak-Thépot J: Cyclin B2 suppresses mitotic failure and DNA re-replication in human somatic cells knocked down for both cyclins B1 and B2. Oncogene 26: 7175-7184, 2007.

24. Kalluri R and Weinberg RA: The basics of epithelial-mesenchymal transition. J Clin Invest 119: 1420-1428, 2009.

25. Thiery JP, Acloque H, Huang RY and Nieto MA: Epithelial-mesenchymal transitions in development and disease. Cell 139: 871-890, 2009.

26. Shiota M, Itsumi M, Takeuchi A, Imada K, Yokomizo A, Kuruma H, Inokuchi J, Tatsugami K, Uchiumi T, Oda Y and Naito S: Crosstalk between epithelial-mesenchymal transition and castration resistance mediated by Twist $1 / A R$ signaling in prostate cancer. Endocr Relat Cancer 22: 889-900, 2015.

27. Liu JH, Wei S, Burnette PK, Gamero AM, Hutton M and Djeu JY: Functional association of TGF-beta receptor II with cyclin B. Oncogene 18: 269-275, 1999.

28. Weng J, Wang J, Hu X, Wang F, Ittmann M and Liu M: PSGR2, a novel G-protein coupled receptor, is overexpressed in human prostate cancer. Int J Cancer 118: 1471-1480, 2006.

29. Kohrman AQ and Matus DQ: Divide or conquer: Cell cycle regulation of invasive behavior. Trends Cell Biol 27: 12-25, 2017.

30. Vihma H, Pruunsild $P$ and Timmusk T: Alternative splicing and expression of human and mouse NFAT genes. Genomics 92: 279-291, 2008

31. Esufali S and Bapat B: Cross-talk between Rac1 GTPase and dysregulated Wnt signaling pathway leads to cellular redistribution of beta-catenin and TCF/LEF-mediated transcriptional activation. Oncogene 23: 8260-8271, 2004

32. Yang F, Li X, Sharma M, Sasaki CY, Longo DL, Lim B and Sun Z: Linking beta-catenin to androgen-signaling pathway. J Biol Chem 277: 11336-11344, 2002.

33. Coussens L, Parker PJ, Rhee L, Yang-Feng TL, Chen E, Waterfield MD, Francke U and Ullrich A: Multiple, distinct forms of bovine and human protein kinase $\mathrm{C}$ suggest diversity in cellular signaling pathways. Science 233: 859-866, 1986.

34. Tirado OM, Maccarthy CM, Fatima N, Villar J, Mateo-Lozano S and Notario V: Caveolin-1 promotes resistance to chemotherapy-induced apoptosis in Ewing's sarcoma cells by modulating PKCalpha phosphorylation. Int J Cancer 126: 426-436, 2010

35. Lønne GK, Cornmark L, Zahirovic IO, Landberg G, Jirström K and Larsson C: PKCalpha expression is a marker for breast cancer aggressiveness. Mol Cancer 9: 76, 2010.

36. Lichner Z, Ding Q, Samaan S, Saleh C, Nasser A, Al-Haddad S, Samuel JN, Fleshner NE, Stephan C, Jung K and Yousef GM: miRNAs dysregulated in association with Gleason grade regulate extracellular matrix, cytoskeleton and androgen receptor pathways. J Pathol 237: 226-237, 2015. 